\title{
An Iterative Hyperelastic Parameters Reconstruction for Breast Cancer Assessment
}

\author{
Hatef Mehrabian $^{1}$, Abbas Samani ${ }^{1,2,3}$ \\ ${ }^{1}$ Department of Electrical \& Computer Engineering, University of Western Ontario, London, ON, \\ Canada \\ ${ }^{2}$ Department of Medical Biophysics, University of Western Ontario, London, ON, Canada \\ ${ }^{3}$ Imaging Research Laboratories, Robarts Research Institute, London, ON, Canada
}

\begin{abstract}
In breast elastography, breast tissues usually undergo large compressions resulting in significant geometric and structural changes, and consequently nonlinear mechanical behavior. In this study, an elastography technique is presented where parameters characterizing tissue nonlinear behavior is reconstructed. Such parameters can be used for tumor tissue classification. To model the nonlinear behavior, tissues are treated as hyperelastic materials. The proposed technique uses a constrained iterative inversion method to reconstruct the tissue hyperelastic parameters. The reconstruction technique uses a nonlinear finite element (FE) model for solving the forward problem. In this research, we applied Yeoh and Polynomial models to model the tissue hyperelasticity. To mimic the breast geometry, we used a computational phantom, which comprises of a hemisphere connected to a cylinder. This phantom consists of two types of soft tissue to mimic adipose and fibroglandular tissues and a tumor. Simulation results show the feasibility of the proposed method in reconstructing the hyperelastic parameters of the tumor tissue.
\end{abstract}

KEYWORDS: Breast Cancer, Elastography, Hyperelactic, Inverse Problem, Regularization

\section{INTRODUCTION}

Elastography is a non-invasive method in which stiffness or strain images of soft tissues are used to detect or classify tumors. It is known that changes in the stiffness of soft tissues are associated with the presence of pathology. In breast cancer, a tumor or a suspicious cancerous growth is normally stiffer than the background normal soft tissue. This forms the basis for the commonly used breast manual palpation technique initially used for breast cancer detection. Manual palpation; however, is not sufficiently sensitive with cases where the tumor is not large enough or is located deep within the breast. In such cases, the tumor cannot be detected by palpation in early stages [1]. Thus more qualitative methods are required to detect the presence of abnormalities. In classic elastography the tissue is assumed to exhibit linear behavior, and using Hooke's law, the tissue's elastic behavior is characterized with only one parameter known as the Young's modulus. In quasi-static elastography, the tissue is stimulated by applying very low frequency external compression. While linear elastic behavior of tissue is expected when very small external compression is applied, soft tissues especially breast tissues deform significantly as a result of even small and inevitable body motions such as chest motion due to respiration. As such, applying small external compressions to ensure linear elastic behavior is problematic due to the presence of other uncontrollable factors, e.g. body motion, which lead to tissue compression comparable to the one resulting from external stimulation. In other words, linear elastic behavior is maintained at the cost of having small signal-to-noise ratio (SNR) of tissue deformation. To address this problem, large external compression is applied to the tissue, which results in large tissue deformation. Furthermore, in breast quasi-static elastography, tissue deformation can be very large due to low stiffness of the tissue and lack of physical constraints. This results in nonlinear behavior of the tissue. At large deformations most tissues exhibit significant strain hardening and the Young's modulus can no longer be considered constant [2-3]. As such linear elasticity is not sufficient to model the tissue deformation, thus hyperelastic models are used in this case. Ignoring Hyperelastic effects generally leads to sub-optimal contrast (stiffer tissues at lower strain are contrasted against softer tissues at higher strain) [4].

Medical Imaging 2008: Physiology, Function, and Structure from Medical Images

edited by Xiaoping P. Hu, Anne V. Clough, Proc. of SPIE Vol. 6916, 69161C, (2008)

1605-7422/08/\$18 - doi: $10.1117 / 12.770971$

Proc. of SPIE Vol. 6916 69161C-1 
Large deformations can increase the deformation SNR of the system [5-6]. If the nonlinear behavior is not considered in the reconstruction algorithm, the elastography image contrast as well as its contrast to noise ratio (CNR) decreases. Nonlinear processing can overcome this limitation [4]. Sinkus et al [7] used MR elastography to show that various breast tissue pathologies exhibit different nonlinear mechanical characteristics. Thus, nonlinear elastography can be used as a highly specific breast cancer diagnosis technique.

The ultimate goal of elasticity imaging, i.e. elastography is to find scalar parameters that describe the elastic behavior of each pixel in the image from local elasticity reconstructions and independent of the boundary conditions [4]. In this investigation we have developed a technique similar to the one proposed by Samani et al [8] for hyperelastic parameter reconstruction. Here, we demonstrate a computational breast phantom study where the phantom consists of three different tissue types: adipose, fibroglandular and tumor tissues. The hyperelastic model of the normal adipose and fibroglandular tissues can be found in the literature [9], and the goal is to reconstruct the model parameters for the tumor tissue.

\section{THEORY}

In this study a nonlinear Finite Element (FE) model of the tissue is used in the forward model of the reconstruction technique. In this FE model, the tissues are assumed to be hyperelastic, which undergo finite deformations. Thus, we used a finite deformation formulation to develop this model. The model assumes that the breast tissue is an incompressible isotropic material. Under static conditions, the equilibrium equation for a tissue undergoing external force is

$$
\frac{\partial \sigma_{i 1}}{\partial x_{1}}+\frac{\partial \sigma_{i 2}}{\partial x_{2}}+\frac{\partial \sigma_{i 3}}{\partial x_{3}}+f_{i}=0 \quad i=1,2,3
$$

where $\sigma$ 's are the Cauchy stress tensor components and $f_{i}$ is the body force in the $\mathrm{x}_{\mathrm{i}}$ direction. The current position of a point $x$ in finite deformation is obtained from adding its displacement $u$ to its corresponding reference position $X$.

$$
x=X+u
$$

The constitutive model of the hyperelastic tissues is represented by a strain energy function. Strain energy function defines the relationship between stresses and strain invariants. These functions are set by a number of coefficients called the hyperelastic parameters. Two different strain energy functions are used in this study. The first one follows the polynomial form widely used in various applications including breast elastography, and the other one follows the Yeoh model, which has been recently used in some biomedical applications. The following equations (3) and (4) represent the polynomial form and Yeoh model respectively:

$$
\begin{gathered}
U=\sum_{i+j=1}^{N} C_{i j}\left(\bar{I}_{1}-3\right)^{i}\left(\bar{I}_{2}-3\right)^{j}, \\
U=\sum_{i=1}^{3} C_{i 0}\left(\bar{I}_{1}-3\right)^{i},
\end{gathered}
$$

where in both equations $C_{i j}$ represents the hyperelastic parameters of the model and $\bar{I}_{1}$ and $\overline{I_{2}}$ are the strain invariants.

The polynomial form with $\mathrm{N}=2$ is most commonly used in rubber modeling but has been recently used in breast tissue modeling [9]. This strain energy function depends only on $I_{1}$ and $I_{2}$ while it is independent of $I_{3}$ to satisfy incompressibility property of the material. Unlike the Polynomial form, the Yeoh strain energy function does not depend 
on $I_{2}$ and is only a function of $I_{1}$. In practice there are several reasons for eliminating $I_{2}$ from the strain energy function (Kawabata, Yamashita (1995) and Kaliske, Rothert (1997) et al [10, 11]), which are:

- The sensitivity of the strain energy function to variations in $I_{2}$ and consequently its dependence on $I_{2}$ terms is generally much smaller than the sensitivity to variations to $I_{1}$.

- It is difficult to measure the influence of $I_{2}$ on the strain energy function.

- Eliminating the $I_{2}$ dependent terms of the strain energy function improves the ability of the model in predicting the tissue behavior.

In this study we use both $I_{2}$ dependent (Polynomial) and $I_{2}$ independent (Yeoh) models.

To constrain the reconstruction process, the hyperelastic parameters are assumed to be uniform throughout the volume of each tissue type. Samani et al [9] proposed a system to calculate the hyperelastic properties of adipose and fibroglandular tissues in laboratory. Their study showed that it is feasible to measure the hyperelastic properties of normal breast tissues. Therefore, in this investigation, we assume that the hyperelastic parameters of the adipose and fibroglandular tissues are known and the goal is to find the parameters of the tumor.

\section{METHOD}

A numerical phantom study was performed on a simplified breast tissue geometry comprised of a hemisphere connected to a cylinder. The phantom's FE mesh was constructed using a transfinite interpolation meshing technique. To avoid having extremely distorted elements, which is the weakness of the transfinite interpolation technique in generating circular shapes, the hemispherical part of the mesh was constructed using the method given in [12]. This mesh with element sets corresponding to three tissue types is shown in Fig.1.

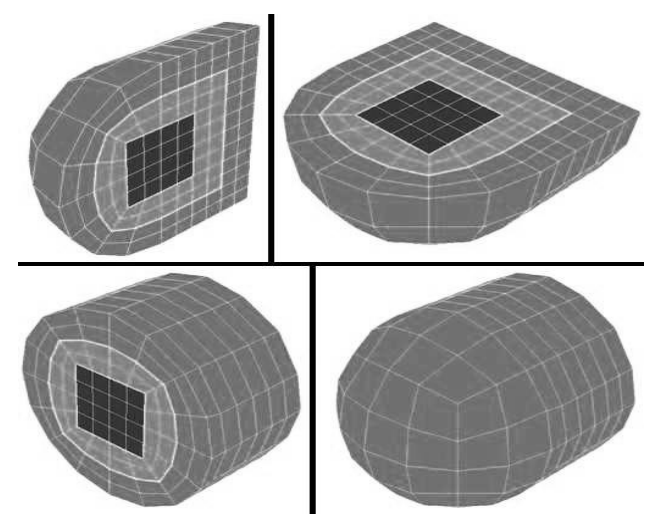

Fig. 1. FE mesh of the computational breast phantom and three corresponding orthogonal cross sections. Different tissue types are shown in different colors, where the interior, middle and exterior layers represent tumor, fibroglandular and adipose tissues, respectively.

The deformation of the tissue is simulated using ABAQUS based on known displacement boundary conditions. The hyperelastic parameters reconstruction technique is iterative and it involves parameter updating followed by FE analysis in each iteration. This parameter reconstruction procedure is summarized in the flowchart shown in Fig.2. 


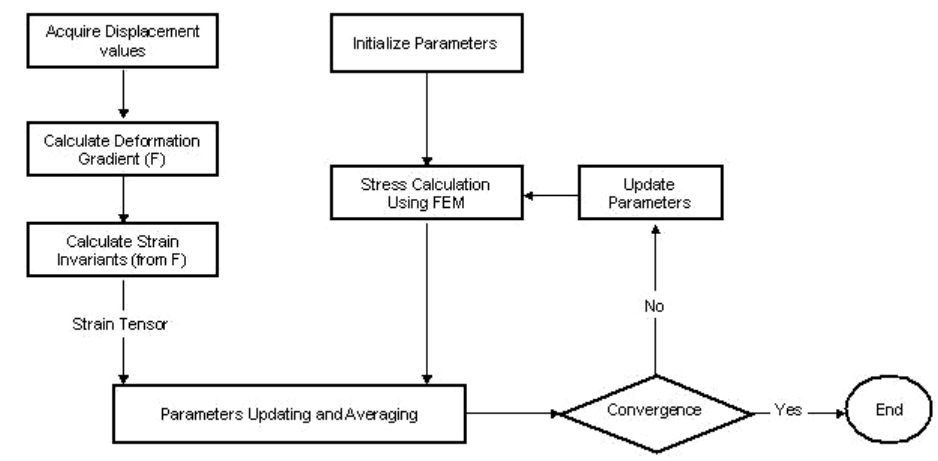

Fig. 2. Flow chart illustrating the procedure of iterative reconstruction of hyperelastic parameters

The iterative process begins with an initial guess for the five (for Polynomial model) or three (for Yeoh model) unknown hyperelastic parameters of the tumor. Using the known boundary conditions, the initial guess and the mesh generated on the segmented tissues, ABAQUS is employed for stress calculation. This is followed by updating the parameters using strain energy function defined in equations (3) or (4) and the stress strain relationship given in equation (5). $[13]$

$$
\sigma=\frac{2}{J} D E V\left[\left(\frac{\partial U}{\partial \overline{I_{1}}}+\overline{I_{1}} \frac{\partial U}{\partial \overline{I_{2}}}\right) \bar{B}-\frac{\partial U}{\partial \overline{I_{2}}} \bar{B} \cdot \bar{B}\right]-p I
$$

where $D E V$ represents the deviatoric part of the stress tensor, $\mathrm{p}$ is hydrostatic pressure, $I$ is the identity matrix and $\bar{B}$ is defined as

$$
\bar{B}=\bar{F} \cdot \bar{F} T
$$

where $F$ is the deformation gradient tensor that describes the displacement of each point during compression. In this equation, $\bar{F}$ is defined as follows for separating the volumetric and deviatoric effects

$$
\begin{aligned}
& \bar{F}=J^{-1 / 3} F, \\
& J=\operatorname{det}(F),
\end{aligned}
$$

This follows the definition of alternate forms of the strain invariants $I_{1}$ and $I_{2}$ as follows:

$$
\begin{aligned}
& \overline{I_{1}}=\operatorname{tr}(\bar{B}), \\
& \overline{I_{2}}=\frac{1}{2}\left({\overline{I_{1}}}^{2}-\operatorname{tr}(\bar{B} \cdot \bar{B})\right),
\end{aligned}
$$

For each element, equation (5) was rearranged in the following form:

$$
\{\sigma\}=[A]\{C\}
$$


Where $\{\sigma\}$ is the element stress tensor, $[A]$ the coefficients matrix formed using nodal displacements and $F$ tensor, and $\{C\}$ is the unknown hyperelastic parameters vector. Using equation (9), the values of $C_{i j}$ were calculated using a least squares method. This yields a set of parameters for each element in the mesh. Averaging these values over the entire volume of the tumor tissue results in the updated parameters of the tissue.

\section{INVERSE PROBLEM}

Solving the inverse problems is done by solving equation (9) using a least squares method. The parameters are calculated using equation (10),

$$
C=\left(A^{T} A\right)^{-1} A^{T} \sigma
$$

this requires calculating the inverse of matrix $\left(A^{T} A\right)$, which is not always possible.

Having five unknown parameters, the Polynomial form with $\mathrm{N}=2$ is widely used for incompressible materials. There is a major problem in using the Polynomial form, which is the difficulty in calculating the inverse of $\left.{ }_{(A}{ }^{T} A\right)$ matrix because of its ill-conditioning. Ill-conditioning becomes an issue in solving a linear system, if any of the following three cases occur.

1. If the determinant of the coefficient matrix is too small

2. If a row/column of the coefficient matrix is close to a linear combination of other rows/columns of the matrix

3. If the ratio of the largest eigenvalue of the coefficient matrix to the smallest one is too large.

All of these cases have similar effect on the system response, which is making it unstable. If a system of equations is ill-conditioned, the round off error in the computations can potentially result in very inaccurate solutions. We have observed that the system of equations for the polynomial form is ill-conditioned. Solutions to this ill-conditioned problem may be found using regularization techniques.

Each regularization technique leads to a certain error in the calculated parameters. To minimize the error, we have developed a regularization technique, which combines three different regularization techniques. The least squares error of the system in each iteration is shown in Fig. 3. In this regularization technique, we divided the iterations range into three regions as shown in the figure. The dashed lines correspond to the first region where the Truncated SVD method was used. The dotted portion of the graph corresponds to the second region where the Tikhonov Regularization technique was used while the last portion of the graph corresponds to the Wiener Filtering regularization technique used for the third iteration region. While the Truncated SVD cannot find the exact solution, it can provide good approximate solutions for systems with large errors. Therefore, it was used in the first set of iterations where errors are expected to be large. When the error is sufficiently small, which is expected in the middle iteration region, Tikhonov regularization was used. For solutions much closer to the exact value of parameters Wiener filtering is used. The Wiener filter used in here regulates the smallest singular value only. Truncated SVD, Tikhonov regularization and Wiener filtering technique are given in equations (11), (12) and (13) respectively.

$$
\begin{aligned}
& A x=b, \quad A^{T} A=U \Sigma V^{T}, \\
& \hat{x}=\sum_{i=1}^{q} \frac{u_{i}^{T} b}{\sigma_{i}} v_{i}, \quad q<\operatorname{rank}\left(A^{T} A\right), \\
& \hat{x}=\left(A^{T} A+\Gamma^{T} \Gamma\right)^{-1}+A^{T} b
\end{aligned}
$$




$$
\hat{x}=\sum_{i=1}^{q} f_{i} \frac{u_{i}^{T} b}{\sigma_{i}} v_{i}, \quad q=\operatorname{rank}\left(A^{T} A\right), f_{i}=\frac{\sigma_{i}^{2}}{\sigma_{i}^{2}+\alpha^{2}}
$$

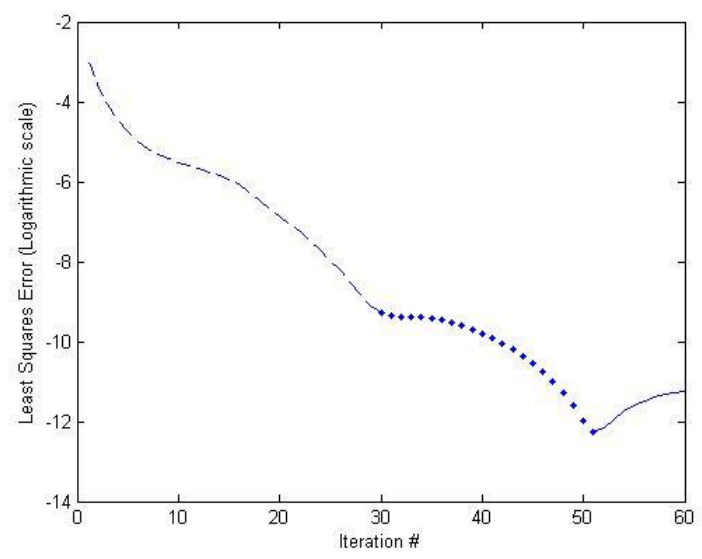

Fig.3. Least squares error of the system at each iteration, The dashed line corresponds to the Truncated SVD method. The dotted and solid lines correspond to the Tikhonov Regularization technique and the Wiener Filtering regularization technique, respectively.

\section{RESULTS}

$30 \%$ compression was applied to the breast phantom. The coefficients matrix was formed using the nodal displacements of the model, derived from the positions of nodal point before and after applying compression. The inverse problem was solved using the iterative optimization method illustrated in Fig.2. While solving the system of equations obtained with the Yeoh model did not require regularization, the system of equations corresponding to the Polynomial model was inverted using the regularization technique we developed. Convergence of each parameter to the corresponding final values is illustrated in Fig.4. These figures indicate that the proposed technique converges to accurate values rapidly for all parameters in both models. The stress-strain relationship corresponding to the true parameter values and those corresponding to the reconstructed parameter values for both Yeoh and Polynomial forms are shown in Fig. 5.

Table 1. shows the initial guess, true parameter values, calculated parameter values, number of iterations required for convergence, the tolerance used in the convergence criteria, and the percentage error of the calculated values. The initial guesses are chosen two to three orders of magnitude larger than the corresponding true values of each parameter to demonstrate the robustness of the proposed method. These results show that convergence to accurate values was achieved after relatively small numbers of iteration. 


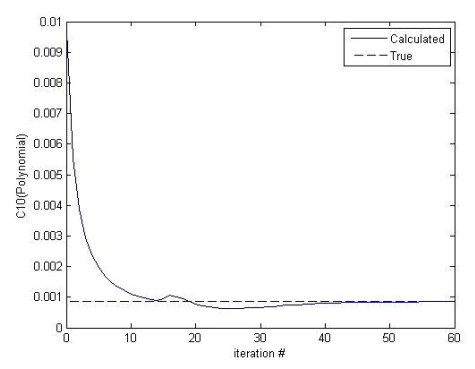

(a)

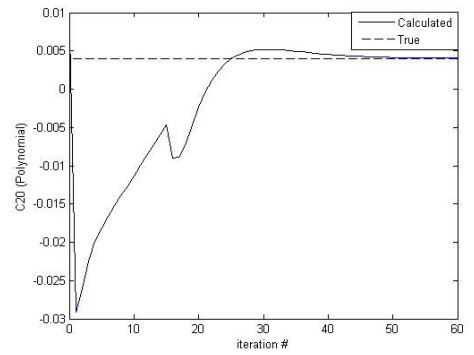

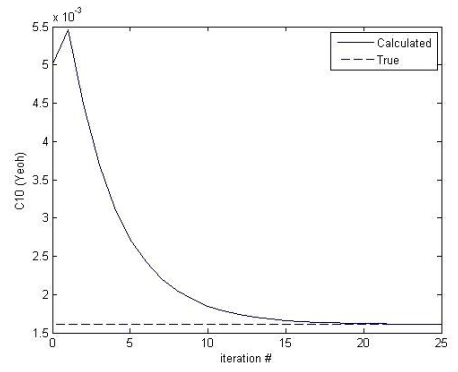

(f)

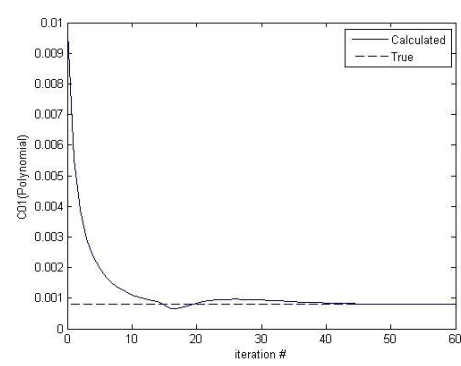

(b)

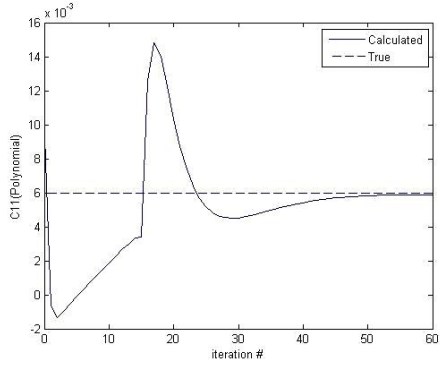

(c) (d)

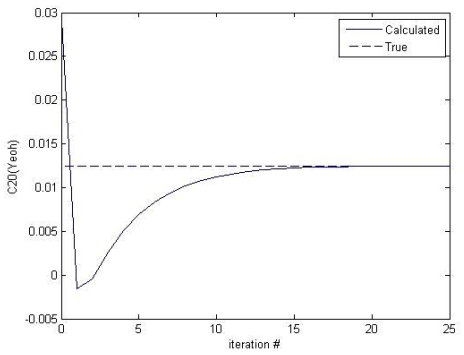

(g)

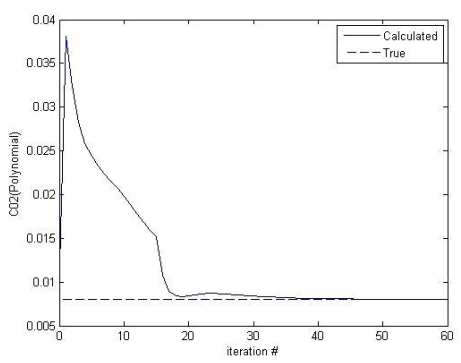

(e)

Fig. 4. a, b, c, d, e) show the convergence of C10, C01, C11, C20, and C02 in the Polynomial form, respectively. f, g, h) show the convergence of $\mathrm{C} 10, \mathrm{C} 20$, and $\mathrm{C} 30$ in Yeoh form respectively.

Table 1. The initial guess, true values of the hyperelastic parameters, calculated values of the parameters, number of iteration required to reach these values, the tolerances used as convergence criteria and the error percentage of the calculated values

\begin{tabular}{|l|c|c|c|c|c|c|}
\hline & $\begin{array}{c}\text { Initial } \\
\text { Guess(kpa) }\end{array}$ & $\begin{array}{c}\text { True } \\
\text { Value(kpa) }\end{array}$ & $\begin{array}{c}\text { Calculated Value } \\
(\mathrm{kpa})\end{array}$ & $\begin{array}{c}\text { Iteration } \\
\text { Number }\end{array}$ & $\begin{array}{c}\text { Tolerance } \\
(\text { tol \%) }\end{array}$ & $\begin{array}{c}\text { Error } \\
(\%)\end{array}$ \\
\hline C10 (Polynomial) & 0.01 & 0.00085 & 0.000849 & 60 & 0.04 & 0.038 \\
\hline C01 (Polynomial) & 0.01 & 0.0008 & 0.000799 & 60 & 0.04 & 0.016 \\
\hline C20 (Polynomial) & 0.01 & 0.004 & 0.004065 & 60 & 0.04 & 1.630 \\
\hline C11 (Polynomial) & 0.01 & 0.006 & 0.005883 & 60 & 0.04 & 1.950 \\
\hline C02(Polynomial) & 0.01 & 0.008 & 0.008051 & 60 & 0.04 & 0.648 \\
\hline
\end{tabular}

\begin{tabular}{|l|l|l|l|l|l|l|}
\hline C10 (Yeoh) & 0.005 & 0.00161 & 0.001612 & 25 & 0.2 & 0.143 \\
\hline C20 (Yeoh) & 0.03 & 0.0125 & 0.012487 & 25 & 0.2 & 0.1 \\
\hline C30 (Yeoh) & 0.01 & 0.00551 & 0.005541 & 25 & 0.2 & 0.563 \\
\hline
\end{tabular}




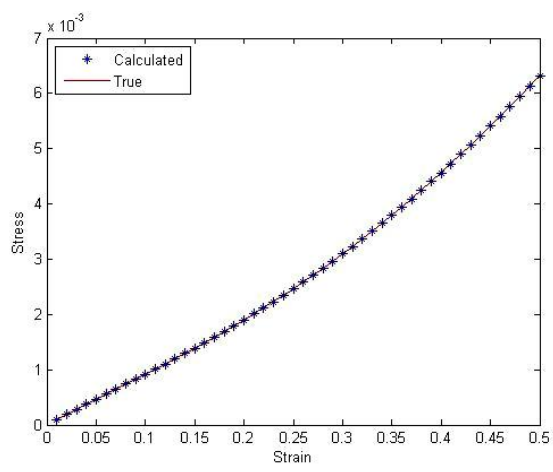

(a)

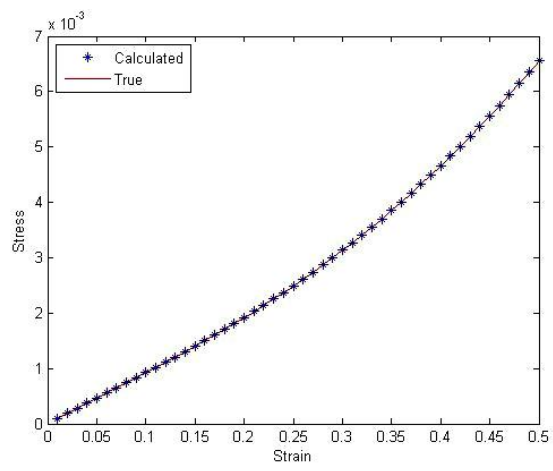

(c)

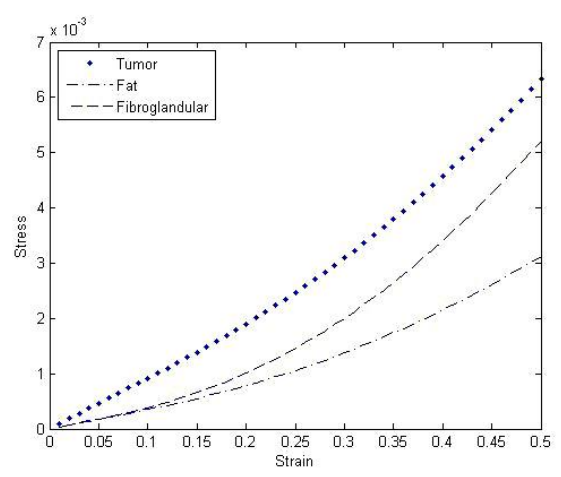

(b)

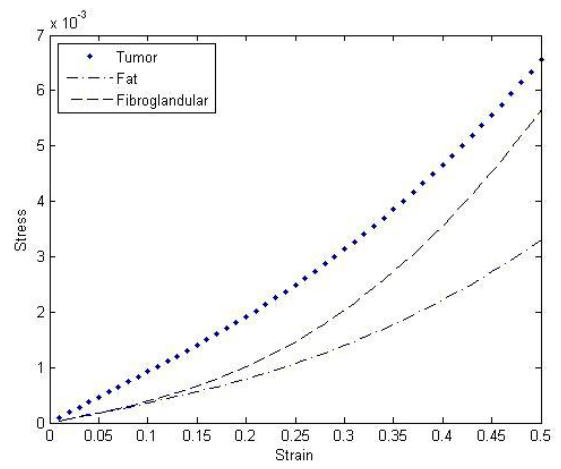

(d)

Fig.5. a) true and reconstructed stress-strain curves of the tumor tissue of the Polynomial form, b) true stress-strain relationship of the fat, fibroglandular and tumor tissues of the Polynomial form, c) true and reconstructed stress-strain curves of the tumor tissue of the Yeoh form, d) true stress-strain relationship of the fat, fibroglandular and tumor tissues of the Yeoh form.

\section{CONCLUSIONS}

In this article a novel nonlinear elastography technique was presented for breast cancer diagnosis. In this method the breast tissue was assumed to be hyperelastic. The proposed technique used an iterative least squares method to find the hyperelastic parameters of the tumor. This method is tested on a simulated breast phantom using two different forms for strain energy function: the Yoeh form and the Polynomial form. The results demonstrated that it is feasible to reconstruct breast tissue hyperelastic parameters from measured displacement data. The Polynomial model depends on strain invariants $I_{1}$ and $I_{2}$ while Yeoh model depends only on one strain invariant $I_{1}$. The Yeoh model converges in less iterations than the Polynomial model with higher accuracy, therefore, eliminating $I_{2}$ dependent terms in the strain energy function results in higher stability in the system. In the future we will use this technique and assess its performance in a breast phantom made of synthetic materials. We will also use the exponential Veronda-Westman strain energy form, which is another commonly used form in modeling the hyperelastic behavior of biological tissues.

\section{REFERENCES}

[1] C. U. Devi, R. S. B. Chandran, R. M. Vasu and A. K. Sood, "Elastic property estimation using ultrasound assisted optical elastography through remote palpation-A simulation study," in 2006. 
[2] F. A. Duck, Physical Properties if Tissues: A Comprehensive Reference Book. London: Academic Press, 1990,

[3] T. A. Krouskop, T. M. Wheeler, F. Kallel, B. S. Garra and T. Hall, "Elastic moduli of breast and prostate tissues under compression," Ultrasonic Imaging, vol. 20, pp. 260-274, 1998.

[4] R. Q. Erkamp, S. Y. Emelianov, A. R. Skovoroda and M. O'Donnell, "Nonlinear elasticity imaging: Theory and phantom study," IEEE Transactions on Ultrasonics, Ferroelectrics, and Frequency Control, vol. 51, pp. 532-539, 2004.

[5] M. O'Donnell, S. Y. Emelianov, A. R. Skovoroda, M. A. Lubinski, W. F. Weitzel and R. C. Wiggins, "Quantitative elasticity imaging," in 1993, pp. 893-903.

[6] T. Varghese and J. Ophir, "Performance optimization in elastography: Multicompression with temporal stretching," Ultrasonic Imaging, vol. 18, pp. 193-214, 1996.

[7] R. Sinkus, S. Weiss, E. Wigger, J. Lorenzen, M. Dargatz and C. Kuhl, "Nonlinear elastic tissue properties of the breast measured by MR-elastography: Initial in-vitro and in-vivo results," Proc. ISMRM 10th Annual Meeting, pp. 33, 2002.

[8] A. Samani, J. Bishop and D. B. Plewes, "A constrained modulus reconstruction technique for breast cancer assessment," IEEE Transactions on Medical Imaging, vol. 20, pp. 877-885, 2001.

[9] A. Samani and D. Plewes, "A method to measure the hyperelastic parameters of ex vivo breast tissue samples," Physics in Medicine and Biology, vol. 49, pp. 4395-4405, 2004.

[10] Y. Yamashita and S. Kawabata, "Longitudinal compression property of wool fiber," Proc. of 24th Text. Research Sympo. at Mt. Fuji, pp. 16-21, 1995.

[11] M. Kaliske and H. Rothert, "On the finite element implementation of rubber-like materials at finite strains," Engineering Computations (Swansea, Wales), vol. 14, pp. 216-232, 1997.

[12] T. J. Horgan and M. D. Gilchrist, "Influence of Fe model variability in predicting brain motion and intracranial pressure changes in head impact simulations," International Journal of Crashworthiness, vol. 9, pp. 401-418, 2004.

[13] G. A. Holzapfel, Nonlinear Solid Mechanics: A Continuum Approach for Engineering. Chichester; New York: Wiley, 2000. 